\title{
Asymptotic Optimality of Equal Power Allocation for Linear Estimation of WSS Random Processes
}

\author{
Boulat A. Bash, Dennis Goeckel, Don Towsley,
}

\begin{abstract}
This letter establishes the asymptotic optimality of equal power allocation for measurements of a continuous wide-sense stationary (WSS) random process with a square-integrable autocorrelation function when linear estimation is used on equally-spaced measurements with periodicity meeting the Nyquist criterion and with the variance of the noise on any sample inversely proportional to the power expended by the user to obtain that measurement.
\end{abstract}

\section{INTRODUCTION}

Linear estimation of a random process from a set of noisy measurements is employed in many wireless communication and sensing applications. We consider a scenario where a continuous

B. A. Bash and D. Towsley are with the Computer Science Department, University of Massachusetts, Amherst, Massachusetts.

D. Goeckel is with the Electrical and Computer Engineering Department, University of Massachusetts, Amherst, Massachusetts.

This research was sponsored by the National Science Foundation under grants CNS-0905349 and CNS-1018464, and by the U.S. Army Research Laboratory and the U.K. Ministry of Defence under Agreement Number W911NF-06-3-0001. The views and conclusions contained in this document are those of the author(s) and should not be interpreted as representing the official policies, either expressed or implied, of the U.S. Army Research Laboratory, the U.S. Government, the U.K. Ministry of Defence or the U.K. Government. The U.S. and U.K. Governments are authorized to reproduce and distribute reprints for Government purposes notwithstanding any copyright notation hereon. 
wide-sense stationary (WSS) random process with a square-integrable autocorrelation function $(\mathrm{ACF})$ is to be estimated using a Wiener filter from measurements that are equally-spaced with periodicity meeting the Nyquist criterion and subject to additive Gaussian white noise (AWGN) with variance inversely proportional to the power expended by the observer to obtain it. A Wiener filter estimator is optimal in the mean squared error sense if the random process is also Gaussian. Generally, if the observation period is finite in this setting, equal power allocation is suboptimal. However, equal power allocation is simple to implement and performs well when the observation period is long enough to reduce the importance of the "edge effects". In this letter we confirm this intuition by proving that the optimal power allocation across equally-spaced measurements indeed tends to equality in the asymptotic case of an infinite number of measurements of a WSS random process with a square-integrable ACF.

Our scenario frequently arises in pilot symbol assisted modulation (PSAM) on wireless channels. A Gaussian random process with a square-integrable ACF often governs the behavior of the wireless channel, ensuring the optimality of Wiener filtering of the channel measurements collected by the receiver from the known pilot signals inserted into the transmission by the sender at the Nyquist rate for the process. Many PSAM techniques correspond to different wireless channel models (see survey [1] and references therein). However, we prove a general result, confirming the intuition behind equally-spaced equal-power pilots used in the original work analyzing PSAM [2] (and similar approaches).

In the following section we formally state our problem. In Section III we establish preliminary results that facilitate the proof of the asymptotic optimality of equal power allocation for linear estimation of WSS random processes in Section IV] Section $\nabla$ concludes the letter.

\section{Power Allocation Problem}

Consider a continuous-time WSS random process $x(t)$ with a square integrable ACF $R_{x}(\tau)$ : $\int_{-\infty}^{\infty}\left|R_{x}(\tau)\right|^{2} d \tau<\infty$. We sample $x(t)$ at rate $\frac{1}{T_{s}}$ that meets the Nyquist criterion, collecting $n$ samples of $x(t)$ from the interval $\left[0,(n-1) T_{s}\right]$. Let $x_{i}^{(n)}=x\left(i T_{s}\right)$. Typically (e.g. for PSAM) the 
discrete observations of $x_{i}^{(n)}$ take the following form: $\tilde{y}_{i}^{(n)}=\sqrt{P_{i}^{(n)}} x_{i}^{(n)}+\tilde{z}_{i}^{(n)}$, where $\left\{\tilde{z}_{i}^{(n)}\right\}$ is an independent and identically distributed (i.i.d.) AWGN sequence with $\tilde{z}_{i}^{(n)} \sim \mathcal{N}\left(0, \sigma^{2}\right)$, and $P_{i}^{(n)}>$ 0 is the power used by the observer for the $i^{\text {th }}$ observation. Normalizing the observations by $\left(P_{i}^{(n)}\right)^{-1 / 2}$ simplifies the analysis without affecting performance, yielding $y_{i}^{(n)}=\tilde{y}_{i}^{(n)} / \sqrt{P_{i}^{(n)}}=$ $x_{i}^{(n)}+z_{i}^{(n)}$, where $\left\{z_{i}^{(n)}\right\}$ is an i.i.d. sequence with $z_{i}^{(n)} \sim \mathcal{N}\left(0, \sigma^{2} / P_{i}\right)$. The observer is subject to the peak power constraint $P_{\max }$ such that $P_{i}^{(n)} \leq P_{\max }$ for all $i$, where $P_{\max }$ is a finite constant. Let $P_{T}(n)=\sum_{i=0}^{n-1} P_{i}^{(n)}$ denote the total power allocated to $n$ observations of the process.

We estimate $x_{i}^{(n)}$ from $y_{i}^{(n)}$ using a Wiener filter over the finite-time horizon $\left[0,(n-1) T_{s}\right]$ for an increasing number of observations $n$. Denote the sequence of covariance matrices of $x_{i}^{(n)}$ as $\left\{\mathbf{R}_{n}\right\}$, where $\left(\mathbf{R}_{n}\right)_{i, j}=R_{x}\left(|i-j| T_{s}\right)$. Since $R_{x}(\tau)$ is square integrable and since $x(t)$ is sampled at the Nyquist rate, the sequence $\left\{R_{x}\left(k T_{s}\right)\right\}_{k=0}^{n-1}$ that forms $\mathbf{R}_{n}$ is square summable1: $\sum_{k=-\infty}^{\infty}\left|R_{x}\left(k T_{s}\right)\right|^{2}<\infty$. Since the noise $z_{i}^{(n)}$ is i.i.d., the sequence of covariance matrices of the observation process is $\left\{\mathbf{R}_{n}+\mathbf{D}_{n}\right\}$, where $\mathbf{D}_{n}=\operatorname{diag}\left(\frac{\sigma^{2}}{P_{0}^{(n)}}, \frac{\sigma^{2}}{P_{1}^{(n)}}, \ldots, \frac{\sigma^{2}}{P_{n-1}^{(n)}}\right)$ defines a sequence of diagonal matrices. Note that the observation process depends on the sequence of power allocation vectors $\left\{\mathbf{p}^{(n)}\right\}$ through $\mathbf{D}_{n}$, where $\mathbf{p}^{(n)}=\left[P_{0}^{(n)}, P_{1}^{(n)}, \ldots, P_{n-1}^{(n)}\right]$. By [4, Eq. (12.53)], $\hat{\mathbf{x}}^{(n)}=\mathbf{R}_{n}\left(\mathbf{R}_{n}+\mathbf{D}_{n}\right)^{-1} \mathbf{y}^{(n)}$ defines the sequence of estimate vectors $\left\{\hat{\mathbf{x}}^{(n)}\right\}$, where the $i^{\text {th }}$ row of matrix $\mathbf{R}_{n}\left(\mathbf{R}_{n}+\mathbf{D}_{n}\right)^{-1}$ contains the Wiener filter coefficients for the estimate of $x_{i}^{(n)}$. Denoting the expectation operator by $\mathbb{E}[\cdot]$ and the transpose of matrix $\mathbf{A}$ by $\mathbf{A}^{T}$, the covariance matrix $\mathbf{M}_{n}=\mathbb{E}\left[\mathbf{e}^{(n)}\left(\mathbf{e}^{(n)}\right)^{T}\right]$ of the estimate error $\mathbf{e}^{(n)}=\mathbf{x}^{(n)}-\hat{\mathbf{x}}^{(n)}$ is [4, Eq. (12.55)]:

$$
\begin{aligned}
\mathbf{M}_{n} & =\mathbf{R}_{n}-\mathbf{R}_{n}\left(\mathbf{R}_{n}+\mathbf{D}_{n}\right)^{-1} \mathbf{R}_{n} \\
& =\left(\mathbf{R}_{n}^{-1}+\mathbf{D}_{n}^{-1}\right)^{-1}
\end{aligned}
$$

where (2) is due to [5, Ch. 0.7.4]. Again, note that the sequence $\left\{\mathbf{M}_{n}\right\}$ depends on the sequence of power allocation vectors $\left\{\mathbf{p}^{(n)}\right\}$ via $\mathbf{D}_{n}$.

\footnotetext{
${ }^{1}$ Since $R_{x}(\tau)$ is square integrable and band-limited to $\frac{1}{2 T_{s}}$, it is in a Hilbert space $\mathcal{H}$. A complete orthonormal basis for such is $\left\{\operatorname{sinc}\left(\tau / T_{s}-k\right)\right\}_{k=-\infty}^{\infty}$ [3, Ch. 3.18.3]. By Parseval's identity [3, Th. 3.5(3)], $\int_{-\infty}^{\infty}\left|R_{x}(\tau)\right|^{2} d \tau=T_{s}^{2} \sum_{k=-\infty}^{\infty}\left|R_{x}\left(k T_{s}\right)\right|^{2}$.
} 
We are interested in the relationship between the power allocation vector $\mathbf{p}^{(n)}$ and the mean squared error (MSE) of the estimate over the entire observation window, as the size of the window, $n$, grows large. Note that the diagonal entries of $\mathbf{M}_{n}$ contain the MSE of each observation. Thus, denoting the trace of the matrix $\mathbf{A}$ by $\operatorname{Tr}[\mathbf{A}]$, the MSE over all observations is:

$$
\mathcal{E}\left(\mathbf{p}^{(n)}\right) \equiv \frac{1}{n} \sum_{i=0}^{n-1} \mathbb{E}\left[e_{i}^{2}\right]=\frac{1}{n} \operatorname{Tr}\left[\mathbf{M}_{n}\right]
$$

Our main result is the following theorem:

Theorem 1. The MSE $\mathcal{E}\left(\mathbf{p}_{\text {opt }}^{(n)}\right)$ of the optimal power allocation $\mathbf{p}_{\text {opt }}^{(n)}$ converges to the MSE $\mathcal{E}\left(\mathbf{p}_{e q}^{(n)}\right)$ of the equal power allocation $\mathbf{p}_{e q}^{(n)}=\left\{P_{i}^{(n)}: P_{i}^{(n)}=P_{e q}\right\}$ as $n \rightarrow \infty$, with $P_{e q}=\frac{P_{T}(n)}{n}$.

In a typical scenario when $P_{T}(n)$ increases linearly with $n, P_{\mathrm{eq}}$ is a constant. In order to prove this theorem in Section IV] we provide several essential lemmas in the next section.

\section{PREREQUISITES}

We first prove that (3) is strictly convex over all choices of power allocation, demonstrating the uniqueness of the optimal power allocation. We then introduce cyclically-symmetric functions, and prove that, if such functions are strictly convex on a convex domain, then the unique vector that attains their minimum has equal values. The section concludes with the introduction of the asymptotic equivalence of Toeplitz and circulant matrices using material from [6] and [7].

\section{A. Power Allocation Vector that Minimizes MSE is Unique}

Lemma 1. If $\mathbf{A}$ is a real symmetric positive-definite $n \times n$ matrix, then the function $f(\mathbf{x})=$ $\operatorname{Tr}\left[(\mathbf{A}+\operatorname{diag}(\mathbf{x}))^{-1}\right]$ is strictly convex within the polytope $\sum_{i=0}^{n-1} x_{i}=C, x_{i}>0$.

Proof: Since the domain of $f(\mathbf{x})$ is convex [8, Ch. 2.1.2 and 2.1.4], $f(\mathbf{x})$ is strictly convex in $\mathbf{x}$ if and only if $g(t)=f(\mathbf{x}+t \mathbf{v})$ is strictly convex in $t$ for any $t \in \mathbb{R}$ and $\mathbf{v} \in \mathbb{R}^{n}$ such that $\mathbf{x}+t \mathbf{v}$ is in the domain of $f(\mathbf{x})$ (i.e. $\mathbf{x}+t \mathbf{v}$ is a real vector with positive entries that sum 
to $C$ ) [8, Ch. 3.1.1]. This follows directly from the definition of convexity and is known as the method of restriction to a line. Define $\mathbf{B} \equiv \mathbf{A}+\operatorname{diag}(\mathbf{x})+t \operatorname{diag}(\mathbf{v})$ and note that it is symmetric positive-definite (as is $\mathbf{B}^{-1}$ ) since $\mathbf{A}$ is symmetric positive-definite and $\operatorname{diag}(\mathbf{x})+t \operatorname{diag}(\mathbf{v})$ is a diagonal matrix with positive entries on the diagonal. Consider $h(t)=\mathbf{u}^{T} \mathbf{B}^{-1} \mathbf{u}$, where $\mathbf{u}$ is an arbitrary non-zero vector. Its first two derivatives with respect to $t$ are [9, Ch. D.2.1]:

$$
\begin{aligned}
h^{\prime}(t) & =-\mathbf{u}^{T} \mathbf{B}^{-1} \operatorname{diag}(\mathbf{v}) \mathbf{B}^{-1} \mathbf{u} \\
h^{\prime \prime}(t) & =2 \mathbf{w}^{T} \mathbf{B}^{-1} \mathbf{w}
\end{aligned}
$$

where $\mathbf{w}=\operatorname{diag}(\mathbf{v}) \mathbf{B}^{-1} \mathbf{u}$. We can substitute $\mathbf{w}$ into (5) since $\mathbf{B}$ is symmetric. Also, since $\mathbf{B}^{-1}$ is positive-definite, $h^{\prime \prime}(t)>0$, implying that $h(t)$ is strictly convex in $t$. Now

$$
\begin{aligned}
g(t) & =\operatorname{Tr}\left[(\mathbf{A}+\operatorname{diag}(\mathbf{x})+t \operatorname{diag}(\mathbf{v}))^{-1}\right] \\
& =\sum_{i=0}^{n-1} \mathbf{e}_{i}^{T}(\mathbf{A}+\operatorname{diag}(\mathbf{x})+t \operatorname{diag}(\mathbf{v}))^{-1} \mathbf{e}_{i}
\end{aligned}
$$

where $\mathbf{e}_{i}$ is a vector containing one in the $i^{\text {th }}$ location and zeros everywhere else. Since each summand of (7) can be written down as $h(t)$ (with $\mathbf{e}_{i}$ replacing $\mathbf{u}$ ) and since the sum preserves convexity, $g(t)$ is strictly convex in $t$. Therefore, $f(\mathbf{x})$ is strictly convex in $\mathbf{x}$.

Since $\mathbf{R}_{n}$ is symmetric positive-definite, so is its inverse. Also $\mathbf{D}_{n}^{-1}=\frac{\operatorname{diag}\left(\mathbf{p}^{(n)}\right)}{\sigma^{2}}$. Thus, by Lemma 1, $\mathcal{E}\left(\mathbf{p}^{(n)}\right)$ is strictly convex and $\mathbf{p}_{\text {opt }}^{(n)}$ that minimizes MSE in our problem is unique.

\section{B. Cyclically-symmetric Functions}

We next introduce a class of symmetric functions and prove a useful property about them.

Definition 1 (Cyclically-symmetric function). $f\left(x_{0}, x_{1}, \ldots, x_{n-1}\right)$ is cyclically-symmetric if

$$
f\left(x_{0}, x_{1}, \ldots, x_{n-1}\right)=f\left(x_{1}, \ldots, x_{n-1}, x_{0}\right)
$$

Lemma 2. Suppose $f\left(x_{0}, x_{1}, \ldots, x_{n-1}\right)$ is strictly convex and cyclically-symmetric on a convex domain $\mathcal{S}$. If vector $\mathbf{x}^{*}=\arg \min _{\mathbf{x} \in \mathcal{S}} f\left(x_{0}, x_{1}, \ldots, x_{n-1}\right)$, then $x_{0}^{*}=x_{1}^{*}=\ldots=x_{n-1}^{*}$. 
Proof: Since $f\left(x_{0}, x_{1}, \ldots, x_{n-1}\right)$ is strictly convex, $\mathbf{x}^{*}$ is unique. Since $f\left(x_{0}, x_{1}, \ldots, x_{n-1}\right)$ is cyclically-symmetric, then, for all $i=1, \ldots, n-1, \mathbf{x}^{*}$ also minimizes $f\left(x_{i}, \ldots, x_{n-1}, x_{1}, \ldots, x_{i-1}\right)$. Thus, $x_{0}^{*}=x_{1}^{*}=\ldots=x_{n-1}^{*}$.

\section{Asymptotically Equivalent Matrices}

Results on the asymptotic equivalence of matrix sequences in [6, Ch. 2] enable the discussion of the Toeplitz and circulant matrices at the end of this section. First, let $\mathbf{A}$ be a real-valued $n \times n$ matrix. Then we define the matrix norms as follows:

Definition 2 (Strong norm). $\|\mathbf{A}\|=\max _{\mathbf{z :}: \mathbf{z}^{T} \mathbf{z}=1}\left[\mathbf{z}^{T} \mathbf{A}^{T} \mathbf{A} \mathbf{z}\right]^{1 / 2}$.

Definition 3 (Weak norm). $|\mathbf{A}|=\sqrt{\frac{1}{n} \operatorname{Tr}\left[\mathbf{A}^{H} \mathbf{A}\right]}$.

If $\mathbf{A}$ is symmetric positive-definite with eigenvalues $\left\{\lambda_{i}\right\}_{i=0}^{n-1},|\mathbf{A}|=\sqrt{\frac{1}{n} \sum_{i=0}^{n-1} \lambda_{i}^{2}}$. Also, $\|\mathbf{A}\|=$ $\lambda_{\max }$ and $\left\|\mathbf{A}^{-1}\right\|=1 / \lambda_{\min }$, where $\lambda_{\max }$ and $\lambda_{\min }$ are the maximum and minimum eigenvalues of $\mathrm{A}$, respectively.

Lemma 3 (Lemma 2.3 in [6]). For $n \times n$ matrices $\mathbf{A}$ and $\mathbf{B},|\mathbf{A B}| \leq\|\mathbf{A}\| \cdot|\mathbf{B}|$.

Now define the asymptotic equivalence of matrix sequences as [6, Ch. 2.3]:

Definition 4 (Asymptotically Equivalent Sequences of Matrices). The sequences of $n \times n$ matrices $\left\{\mathbf{A}_{n}\right\}$ and $\left\{\mathbf{B}_{n}\right\}$ are said to be asymptotically equivalent if the following hold:

$$
\begin{gathered}
\left\|\mathbf{A}_{n}\right\|,\left\|\mathbf{B}_{n}\right\| \leq M<\infty, n=1,2, \ldots \\
\lim _{n \rightarrow \infty}\left|\mathbf{A}_{n}-\mathbf{B}_{n}\right|=0
\end{gathered}
$$

We abbreviate the asymptotic equivalence of the sequences $\left\{\mathbf{A}_{n}\right\}$ and $\left\{\mathbf{B}_{n}\right\}$ by $\mathbf{A}_{n} \sim \mathbf{B}_{n}$. Properties of asymptotic equivalence are stated and proved in [6, Theorem 2.1]. A property particularly useful in the proof of Theorem 1 is re-stated here as a lemma: 
Lemma 4. If $\mathbf{A}_{n} \sim \mathbf{B}_{n}$ and $\left\|\mathbf{A}_{n}^{-1}\right\|,\left\|\mathbf{B}_{n}^{-1}\right\| \leq K<\infty, n=1,2, \ldots$, then $\mathbf{A}_{n}^{-1} \sim \mathbf{B}_{n}^{-1}$.

Proof: $\left|\mathbf{A}_{n}^{-1}-\mathbf{B}_{n}^{-1}\right|=\left|\mathbf{B}_{n}^{-1} \mathbf{B}_{n} \mathbf{A}_{n}^{-1}-\mathbf{B}_{n}^{-1} \mathbf{A}_{n} \mathbf{A}_{n}^{-1}\right| \leq\left\|\mathbf{B}_{n}^{-1}\right\| \cdot\left\|\mathbf{A}_{n}^{-1}\right\| \cdot\left|\mathbf{A}_{n}-\mathbf{B}_{n}\right| \underset{n \rightarrow \infty}{\longrightarrow} 0$ where the inequality is due to Lemma 3 . Another important consequence of asymptotic equivalence follows from [6, Corollary 2.1]:

Lemma 5. If $\mathbf{A}_{n} \sim \mathbf{B}_{n}$, then $\lim _{n \rightarrow \infty} \frac{1}{n} \operatorname{Tr}\left[\mathbf{A}_{n}\right]=\lim _{n \rightarrow \infty} \frac{1}{n} \operatorname{Tr}\left[\mathbf{B}_{n}\right]$ when either limit exists.

Proof sketch: By the Cauchy-Schwarz inequality, $\left|\frac{\operatorname{Tr}\left[\mathbf{A}_{n}-\mathbf{B}_{n}\right]}{n}\right| \leq\left|\mathbf{A}_{n}-\mathbf{B}_{n}\right| \underset{n \rightarrow \infty}{\longrightarrow} 0$.

\section{Sequences of Toeplitz and Circulant Matrices}

An $n \times n$ Toeplitz matrix $\mathbf{T}_{n}$, illustrated in Fig. 1(a), is defined by a sequence $\left\{t_{k}^{(n)}\right\}$ where $\left(\mathbf{T}_{n}\right)_{i, j}=t_{i-j}$. The covariance matrix $\mathbf{R}_{n}$ in Section $\amalg$ is Toeplitz and symmetric. An $n \times n$ circulant matrix $\mathbf{C}_{n}$, illustrated in Fig. 1(b), is defined by a sequence $\left\{c_{k}^{(n)}\right\}$ where $\left(\mathbf{C}_{n}\right)_{i, j}=$ $c_{(j-i) \bmod n}^{(n)}$. Since the sequence $\left\{R_{x}\left(k T_{s}\right)\right\}_{k=0}^{n-1}$ that defines $\mathbf{R}_{n}$ is square summable, we can define an asymptotically equivalent sequence of circulant matrices $\mathbf{C}_{n} \sim \mathbf{R}_{n}$ using [7, Eq. (7)]:

$$
c_{k}^{(n)}=R_{x}\left(k T_{s}\right)+\frac{k}{n}\left(R_{x}\left((n-k) T_{s}\right)-R_{x}\left(k T_{s}\right)\right)
$$

The resulting circulant matrix $\mathbf{C}_{n}$ is symmetric since, by $(\underline{10}), c_{k}^{(n)}=c_{n-k}^{(n)}$.

$$
\mathbf{T}_{n}=\left[\begin{array}{cccc}
t_{0}^{(n)} & t_{-1}^{(n)} & \cdots & t_{-(n-1)}^{(n)} \\
t_{1}^{(n)} & t_{0}^{(n)} & \cdots & t_{-(n-2)}^{(n)} \\
\vdots & & \ddots & \vdots \\
t_{n-1}^{(n)} & t_{n-2}^{(n)} & \cdots & t_{0}^{(n)}
\end{array}\right]
$$

(a) Toeplitz matrix

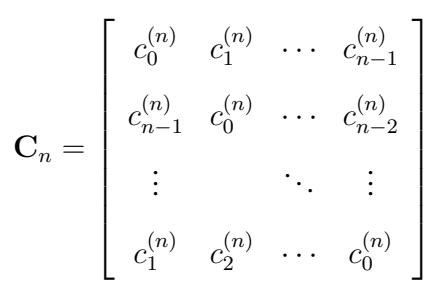

(b) Circulant matrix

Fig. 1. Illustration of Toeplitz and circulant matrices.

By [7, Eq. (5)], $\mathbf{C}_{n} \triangleq \mathbf{F}_{n}^{-1} \boldsymbol{\Delta}_{n} \mathbf{F}_{n}$, where $\boldsymbol{\Delta}_{n}=\operatorname{diag}\left(\left\{\nu_{i}^{(n)}\right\}_{i=0}^{n-1}\right)$ contains the diagonal entries $\nu_{i}^{(n)}=\left(\mathbf{F}_{n} \mathbf{R}_{n} \mathbf{F}_{n}^{-1}\right)_{i, i}$ of the covariance matrix of the discrete Fourier transform (DFT) 
$\mathbf{F}_{n} \mathbf{x}^{(n)}$ of $\mathbf{x}^{(n)}$ and $\left(\mathbf{F}_{n}\right)_{i, k}=\frac{1}{\sqrt{n}} e^{2 \pi i k j / n}$ is the DFT rotation matrix. Since $\mathbf{R}_{n}$ is positive-definite, by the properties of the similarity transformation, $\mathbf{F}_{n} \mathbf{R}_{n} \mathbf{F}_{n}^{-1}$ is positive-definite and has positive diagonal entries. Thus, $\boldsymbol{\Delta}_{n}$ is positive-definite and so is $\mathbf{C}_{n}$.

\section{Asymptotic Optimality of Equal Power Allocation}

Proof (Theorem 11): Define symmetric circulant matrix $\mathbf{C}_{n}$ as in Section III-D so that $\mathbf{C}_{n} \sim \mathbf{R}_{n}$ and consider (2). Asymptotic equivalence results in the following chain of implications:

$$
\begin{aligned}
\mathbf{C}_{n} \sim \mathbf{R}_{n} & \Rightarrow \mathbf{C}_{n}^{-1} \sim \mathbf{R}_{n}^{-1} \\
& \Rightarrow \mathbf{C}_{n}^{-1}+\mathbf{D}_{n}^{-1} \sim \mathbf{R}_{n}^{-1}+\mathbf{D}_{n}^{-1} \\
& \Rightarrow\left(\mathbf{C}_{n}^{-1}+\mathbf{D}_{n}^{-1}\right)^{-1} \sim\left(\mathbf{R}_{n}^{-1}+\mathbf{D}_{n}^{-1}\right)^{-1} \\
& \Rightarrow \lim _{n \rightarrow \infty} \frac{1}{n} \operatorname{Tr}\left[\mathbf{L}_{n}\right]=\lim _{n \rightarrow \infty} \frac{1}{n} \operatorname{Tr}\left[\mathbf{M}_{n}\right]
\end{aligned}
$$

where $\mathbf{L}_{n} \equiv\left(\mathbf{C}_{n}^{-1}+\mathbf{D}_{n}^{-1}\right)^{-1}$. Since $\mathbf{R}_{n}$ and $\mathbf{C}_{n}$ are symmetric positive-definite, the conditions for Lemma 4 hold, resulting in (11). Then (12) follows from adding $\mathbf{D}_{n}^{-1}$ to both sides of the asymptotic equivalence relation and noting that the condition (8) is satisfied via Weyl's inequality [5. Theorem 4.3.1] since the peak power constraint on the observer implies that $\mathbf{D}_{n}^{-1}$ has finite eigenvalues. Lemma 4 yields (13), and (14) is due to Lemma 5. Let

$$
\mathcal{E}_{\text {equiv }}\left(\mathbf{p}^{(n)}\right) \equiv \frac{1}{n} \operatorname{Tr}\left[\left(\mathbf{C}_{n}^{-1}+\mathbf{D}_{n}^{-1}\right)^{-1}\right]
$$

Then, since we defined $\mathcal{E}\left(\mathbf{p}^{(n)}\right) \equiv \frac{1}{n} \operatorname{Tr}\left[\mathbf{M}_{n}\right]$ in (3), (14) can be restated as follows:

$$
\lim _{n \rightarrow \infty} \mathcal{E}\left(\mathbf{p}^{(n)}\right)=\lim _{n \rightarrow \infty} \mathcal{E}_{\text {equiv }}\left(\mathbf{p}^{(n)}\right)
$$

Since $\mathbf{C}_{n}$ is symmetric positive-definite, by Lemma 1, $\mathcal{E}_{\text {equiv }}\left(\mathbf{p}^{(n)}\right)$ is strictly convex in $\mathbf{p}^{(n)}$. Showing that $\mathcal{E}_{\text {equiv }}\left(\mathbf{p}^{(n)}\right)$ is cyclically-symmetric with respect to $\mathbf{p}^{(n)}$ would complete the proof by Lemma 2. The discussion of the convergence of $\mathcal{E}\left(\mathbf{p}^{(n)}\right)$ to $\mathcal{E}_{\text {equiv }}\left(\mathbf{p}^{(n)}\right)$ follows the proof.

Denote the similarity transformation $\mathcal{R}_{i}(\mathbf{A}) \triangleq \mathbf{S}_{i} \mathbf{A S}_{i}^{-1}$ of an $n \times n$ matrix $\mathbf{A}$ the rotation of degree $i$, where $\mathbf{S}_{i}=\left[\begin{array}{cc}\mathbf{0} & \mathbf{I}_{(n-i) \times(n-i)} \\ \mathbf{I}_{i \times i} & \mathbf{0}\end{array}\right]$ and $\mathbf{I}_{n \times n}$ is an $n \times n$ identity matrix. $\mathbf{S}_{i}$ is 
a permutation matrix, and is thus orthogonal, implying that $\mathbf{S}_{i}^{-1}=\mathbf{S}_{i}^{T}=\mathbf{S}_{n-i}$. Suppose that the rows and columns of matrix $\mathbf{A}$ are labeled $0, \ldots, n-1$ top-to-bottom and right-to-left, respectively. Then $\mathbf{S}_{i} \mathbf{A}$ produces a matrix with the top $i$ rows of $\mathbf{A}$ shifted to the bottom (i.e. rows $0, \ldots, i-1$ become rows $n-i-1, \ldots, n-1)$, and $\mathbf{A S}_{i}^{-1}$ produces a matrix with the left $i$ columns of $\mathbf{A}$ shifted to the right (i.e. columns $0, \ldots, i-1$ become columns $n-i-1, \ldots, n-1$ ). Shifting the top $i$ rows of a circulant matrix $\mathbf{C}$ down produces the same matrix as shifting the left $n-i$ columns to the right. Thus, $\mathbf{S}_{i} \mathbf{C}=\mathbf{C S}_{i}$, which implies the rotation invariance of circulant matrices: $\mathcal{R}_{i}(\mathbf{C})=\mathbf{C}$.

Inverse $\mathbf{A}^{-1}$ of matrix $\mathbf{A}$ can be expressed as $\left(\mathbf{A}^{-1}\right)_{i, j}=\frac{(-1)^{i+j}}{\operatorname{det}(\mathbf{A})} \mathcal{M}_{j, i}(\mathbf{A})$ where $\operatorname{det}(\mathbf{A})$ denotes the determinant of $\mathbf{A}$ and $\mathcal{M}_{i, j}(\mathbf{A})=\operatorname{det}\left(\mathbf{A}^{(i, j)}\right)$ with the sub-matrix $\mathbf{A}^{(i, j)}$ formed by removing row $i$ and column $j$ from $\mathbf{A}$ [5, Ch. 0.8.1]. Thus, (15) can be re-stated as:

$$
\mathcal{E}_{\text {equiv }}\left(\mathbf{p}^{(n)}\right)=\frac{\sum_{k=0}^{n-1} \mathcal{M}_{k, k}\left(\mathbf{C}_{n}^{-1}+\mathbf{D}_{n}^{-1}\right)}{n \operatorname{det}\left(\mathbf{C}_{n}^{-1}+\mathbf{D}_{p}^{-1}\right)}
$$

The inverse of a circulant matrix, if it exists, is circulant2 [6, Theorem 3.1 (3)]. Due to the rotational invariance of circulant matrices, for all $i=1, \ldots, n-1, \mathcal{R}_{i}\left(\mathbf{C}_{n}^{-1}+\mathbf{D}_{n}^{-1}\right)=$ $\mathbf{C}_{n}^{-1}+\mathcal{R}_{i}\left(\mathbf{D}_{n}^{-1}\right)$ with $\mathcal{R}_{i}\left(\mathbf{D}_{n}^{-1}\right)=\operatorname{diag}\left(\frac{P_{i}^{(n)}}{\sigma^{2}}, \ldots, \frac{P_{n-1}^{(n)}}{\sigma^{2}}, \frac{P_{0}^{(n)}}{\sigma^{2}}, \ldots, \frac{P_{i-1}^{(n)}}{\sigma^{2}}\right)$. The denominator of (17) is cyclically-symmetric with respect to $\mathbf{p}^{(n)}$ due to the rotation being a similarity transformation, which preserves the determinant. Since the submatrix of $\mathbf{C}_{n}^{-1}+\mathbf{D}_{n}^{-1}$ with row $k$ and column $k$ removed is a submatrix of $\mathbf{C}_{n}^{-1}+\mathcal{R}_{i}\left(\mathbf{D}_{n}^{-1}\right)$ with row $(k-i) \bmod n$ and column $(k-i) \bmod n$ removed, the numerator of (17) is also cyclically-symmetric with respect to $\mathbf{p}^{(n)}$. Therefore, $\mathcal{E}_{\text {equiv }}\left(\mathbf{p}^{(n)}\right)$ is cyclically-symmetric and, by Lemma 2, $\arg \min _{\mathbf{p}^{(n)}} \mathcal{E}_{\text {equiv }}\left(\mathbf{p}^{(n)}\right)=\mathbf{p}_{\text {eq }}^{(n)}$. By (16), $\lim _{n \rightarrow \infty} \mathcal{E}\left(\mathbf{p}_{\text {eq }}^{(n)}\right)=\lim _{n \rightarrow \infty} \mathcal{E}_{\text {equiv }}\left(\mathbf{p}_{\text {eq }}^{(n)}\right)$, completing the proof.

Convergence: First bound $\left|\mathcal{E}\left(\mathbf{p}^{(n)}\right)-\mathcal{E}_{\text {equiv }}\left(\mathbf{p}^{(n)}\right)\right|=\left|\frac{1}{n} \operatorname{Tr}\left[\mathbf{L}_{n}-\mathbf{M}_{n}\right]\right| \leq\left|\mathbf{L}_{n}-\mathbf{M}_{n}\right|$ using the Lemma 5 proof idea. Now, as done in the proof of Lemma 4, apply Lemma 3, $\left|\mathbf{L}_{n}-\mathbf{M}_{n}\right| \leq$ $\left\|\mathbf{L}_{n}\right\| \cdot\left\|\mathbf{M}_{n}\right\| \cdot\left\|\mathbf{C}_{n}^{-1}\right\| \cdot\left\|\mathbf{R}_{n}^{-1}\right\| \cdot\left|\mathbf{C}_{n}-\mathbf{R}_{n}\right|$. The strong norm terms are bounded by Weyl's

\footnotetext{
${ }^{2}$ We note that, in general, the inverses of Toeplitz matrices are not Toeplitz.
} 
theorem per the arguments following (14), while the discussion following [7, Eq. (10)] asserts that $\left|\mathbf{C}_{n}-\mathbf{R}_{n}\right| \leq \frac{C}{\sqrt{n}}+\epsilon$ where $C$ and $\epsilon$ are constants, with $\epsilon$ arbitrarily small. Thus, in (16), $\mathcal{E}\left(\mathbf{p}^{(n)}\right)$ and $\mathcal{E}_{\text {equiv }}\left(\mathbf{p}^{(n)}\right)$ converge at a rate proportional to $\frac{1}{\sqrt{n}}$.

Finally, while the numerical results are not in the scope of this letter, our evaluations show that $\mathcal{E}\left(\mathbf{p}_{\text {eq }}^{(n)}\right)$ converges to $\mathcal{E}\left(\mathbf{p}_{\text {opt }}^{(n)}\right)$ fairly quickly 3 in typical wireless communication scenarios.

\section{CONCLUSION}

The asymptotic optimality is established for the equal power allocation between equally-spaced measurements used for Wiener filter estimation of a continuous WSS random process with a square-integrable ACF, where the periodicity of the measurements meets the Nyquist criterion and the measurements are subject to AWGN with variance inversely proportional to the power expended by the observer.

\section{REFERENCES}

[1] L. Tong, B. Sadler, and M. Dong, "Pilot-assisted wireless transmissions: general model, design criteria, and signal processing," IEEE Signal Processing Magazine, vol. 21, no. 6, pp. 12-25, Nov. 2004.

[2] J. K. Cavers, "An analysis of pilot symbol assisted modulation for rayleigh fading channels [mobile radio]," IEEE Transactions on Vehicular Technology, vol. 40, no. 4, pp. 686-693, Nov. 1991.

[3] T. K. Moon and W. C. Stirling, Mathematical Methods and Algorithms for Signal Processing. Upper Saddle River, NJ: Prentice Hall, 2000.

[4] S. M. Kay, Fundamentals of Statistical Signal Processing, Volume I: Estimation Theory, 1st ed. Upper Saddle River, NJ: Prentice Hall, 1993.

[5] R. A. Horn and C. R. Johnson, Matrix Analysis. Cambridge University Press, 1985.

[6] R. M. Gray, "Toeplitz and circulant matrices: A review," Foundations and Trends in Communications and Information Theory, vol. 2, no. 3, pp. 155-239, 2006. [Online]. Available: http://www-ee.stanford.edu/ gray/toeplitz.pdf

${ }^{3}$ E.g.: We used MATLAB R2011a fmincon at default settings with $P_{T}(n)=n$ and $\sigma^{2}=1$, initialized by $\mathbf{p}_{\text {eq }}^{(n)}$, to find $\mathcal{E}\left(\mathbf{p}_{\text {opt }}^{(n)}\right)$ for $R_{x}(\tau)=e^{-|\tau|}$, where $x(t)$ is sampled at the rate $\frac{\tan \frac{0.99 \pi}{2}}{\pi} \mathrm{Hz}$ (capturing $99 \%$ of the spectrum). This converged (i.e. fmincon was not able to improve $\mathcal{E}\left(\mathbf{p}_{\text {opt }}^{(n)}\right)$ below the initial $\mathcal{E}\left(\mathbf{p}_{\mathrm{eq}}^{(n)}\right)$ within the default tolerance $\left.1 \times 10^{-6}\right)$ at $n=84$. For Jakes' model with $R_{x}(\tau)=J_{0}\left(2 \pi f_{D} \tau\right)$ sampled at the rate $2 f_{D} \mathrm{~Hz}$ (Nyquist), convergence was immediate (at $n=1$ ). 
[7] J. Pearl, "On coding and filtering stationary signals by discrete fourier transforms," IEEE Transactions on Information Theory, vol. 19, no. 2, pp. 229-232, Mar. 1973.

[8] S. Boyd and L. Vandenberghe, Convex Optimization. New York, NY, USA: Cambridge University Press, 2004.

[9] J. Dattorro, Convex Optimization \& Euclidean Distance Geometry. Palo Alto, CA: Meeboo Publishing USA, 2005. 